\title{
A Novel Method to Risk Stratify Patients Undergoing Exercise Stress Echocardiography Using a Set of Combined Criteria
}

\author{
Christopher B. Wong, MBBS, FACC ${ }^{1}$ Sue Ellen Leung, RN ${ }^{1}$ Sasa Fukuyanagi, BA, BCM ${ }^{1}$ \\ ${ }^{1}$ Quality Healthcare Medical Service, Hong Kong, China \\ Address for correspondence Christopher B. Wong, Quality Healthcare \\ Int J Angiol 2015;24:35-40. \\ Medical Service, Tower 1, 16th floor, Admiralty Center, Harcourt Road, \\ Hong Kong, China (e-mail: cbwong2007@yahoo.com).
}

Abstract
Keywords
- atherosclerosis
- cardiovascular disease
- coronary artery
- hypercholesterolemia
- hypertension
- ischemia
- PCl

Background and Purposes A novel method using quantitative long-axis function and tissue Doppler in addition to wall motion analysis in exercise stress echocardiography was evaluated. We hypothesized that the novel criteria added additional accuracy in stress echocardiography.

Methods Patients with chest pain and at low-to-intermediate risk for obstructive coronary artery disease (CAD) were retrospectively studied. They underwent stress echocardiography with attention to wall motion abnormalities, left ventricular long-axis function, and tissue Doppler measurement.

Results The results showed that the combined novel criteria (i.e., classifying a case as positive if three out of the following four criteria were fulfilled: (1) abnormal segmental wall motion shortly after peak stress; (2) Ee wave after peak stress less than $10 \mathrm{~cm} / \mathrm{s}$ and Ee/Aa ratio after peak stress less than 1; (3) Sm wave after peak stress less than $10.5 \mathrm{~cm} / \mathrm{s}$; (4) abnormal long-axis left ventricular function) offered a better accuracy for predicting obstructive CAD and future revascularization with a high sensitivity (100\%) and high negative predictive value (100\%) .

Conclusion From a practical standpoint, the combined novel criteria may be useful in improving the diagnostic accuracy of stress echocardiography.
Coronary artery disease (CAD) is a leading cause of death in the Western world. The incidence has been increasing recently in Asian countries. It is important to screen for significant $\mathrm{CAD}$ and to achieve preventive and therapeutic measures as soon as possible. Clinical studies have shown that exercise stress echocardiography has an acceptable sensitivity (80-85\%) and specificity (85-90\%) $)^{1-4}$ in detecting significant CAD. But stress echocardiography is subject to observers' bias and experience, particularly with single-vessel CAD. With suboptimal echocardiographic windows, the interpretation is even more challenging, especially after a vigorous exercise stress test. However, patients with significant deconditioning and suboptimal exercise tolerance often render false-negative results. Past studies ${ }^{5}$ showed that abnormal long-axis function of left ventricle (LV) occurs early in ischemia, sometimes in the absence of regional wall motion. Quantitatively, abnormal long-axis function may be more accurate than wall motion analysis in the presence of single-vessel CAD. In addition, it is possible to measure long-axis function even in subjects with suboptimal image quality which prevents adequate wall motion analysis. In recent years, the technique of tissue Doppler imaging (TDI) ${ }^{6,7}$ has been used as a modality for assessing systolic and diastolic left ventricular performance. Investigators have also used TDI to measure resting or poststress velocities of various myocardial segments of the LV or mitral annulus as an adjunct tool in the diagnosis of obstructive CAD. Therefore, we hypothesized that the use of a set of novel combined criteria using quantitative long-axis function and tissue Doppler in addition to wall motion analysis provided additional accuracy in stress 
echocardiography. Furthermore, we also hypothesized that a short term clinical follow-up of the patients with a negative stress echocardiographic test was associated with a very low major adverse cardiac event (MACE) in a community setting.

\section{Methods}

A retrospective analysis of 100 consecutive patients from a community clinic was conducted, mean age $48 \pm 10$ (range 24-78), 65 of whom were male. These patients presented with chest pain and were at a low-to-intermediate risk for obstructive CAD. Patients who had recent myocardial infarction, unstable angina, uncontrolled hypertension, or established CAD were excluded. They were studied with stress echocardiography between January 2013 and July 2013. Briefly, patients underwent a symptom-limited treadmill exercise according to the Bruce protocol (General Electric Case Treadmill Machine, Palatine, IL). Twelve-lead electrocardiogram (ECG) was obtained just before treadmill exercise for baseline, and continuously recorded thereafter during exercise testing, including the recovery phase. In the first 30seconds of recovery phase, echocardiography (using Philips Machine HD7 XE, Andover, MA) was performed on parasternal long and shortaxes, apical four-, two-, and threechamber views in left lateral position. Subsequent to that, measurement of the long-axis function, tissue Doppler of the mitral annulus, and cardiac dimensions was obtained. Toward the fifth minute of recovery, rest echocardiogram was obtained one more time on the above views. Abnormal regional wall motion of postexercise images in more than two consecutive segments was considered as a positive stress echocardiographic testing (by wall motion criteria).

\section{Long-Axis Function}

Two-dimensional echocardiographic $M$ mode recordings were obtained from apical four-chamber views with the cursor placed at the septal side of the mitral annulus. Recordings were made at the end of the peak exercise at a sweep speed of $50 \mathrm{~mm} / \mathrm{s}$. The amplitude of long-axis shortening (cm) was defined as the maximum excursion of the mitral annulus during systole. Shortening of less than $1.55 \mathrm{~cm}$ in male and $1.35 \mathrm{~cm}$ in female was considered as abnormal.

\section{Tissue Doppler}

Simplified tissue Doppler was obtained at the end of peak exercise in the pulsed wave Doppler mode. Images were analyzed by a single investigator (C.W.). The gain and filters were adjusted to eliminate background noises. The signals were obtained using a sweep speed of $50 \mathrm{~mm} / \mathrm{s}$. From the apical four-chamber view, sample volumes were placed at the septal corner of the mitral annulus. Measurements were made on peak systolic velocities (Sm), early (Ea) and late (Aa) diastolic velocities at the mitral annulus. Measurements were done in three to five cardiac cycles and averaged.

\section{CT Coronary Angiography}

A total of 25 patients underwent 64-slice spiral CT coronary angiography. ECG gating was used for enhanced scan.
Seventy-five to $100 \mathrm{~mL}$ of nonionic contrast agent was injected into the antecubital vein at the flow rate of 4.0 to $5.0 \mathrm{~mL} / \mathrm{s}$ by a high-pressure syringe, which was followed by the injection of $30 \mathrm{~mL}$ saline at the same rate. The scan time was determined by automatic triggering. The best ECG window was selected to perform volume rendering, multiplanar reformation, and maximum intensity projection reformation. The stenosis degrees of involved vessels were analyzed by an experienced radiologist. A diameter stenosis of $>50 \%$ of a coronary artery was considered significant and warranted further investigation with an invasive coronary angiogram.

\section{Coronary Angiography}

Coronary angiography was done in 13 patients via the radial or femoral approach using the Judkins technique. Angiograms were reported by an experienced observer. Significant disease was defined as $>70 \%$ diameter stenosis in a major epicardial coronary artery by visual estimation.

Borderline lesions were further interrogated by pressure flow wire for fractional flow reserve \pm intravascular ultrasound technique. Further details of the above technique may be obtained in published literature..$^{8,9}$

Clinical follow-up was obtained on all patients for the subsequent 6 months. Telephone follow-up was obtained if the patients elected not to return to the clinic. Attempts were made to document any occurrence of MACEs, including allcause death, myocardial infarction, acute coronary syndrome, and a need for revascularization.

\section{Statistical Analysis}

Data were expressed as mean \pm standard deviation for continuous variables and as numbers (percent) for categorical variables.

The differences in continuous variables between patients were analyzed by unpaired Student's $t$-test, and in categorical variables by the Chi-square test.

A probability value of $<0.05$ was considered statistically significant.

\section{Results}

Data from 100 patients with exercise stress echocardiography and subsequent follow-up were analyzed. Demographics were presented in -Table 1 . The mean $( \pm$ SD) patient age was $48 \pm 10$ years (range 24-78). The cohort represented a low-to-intermediate risk group typically encountered in a community cardiac clinic with risk factors as hypertension (58\%) and dyslipidemia (62\%). There was a low prevalence of diabetes mellitus (5\%) and family history of atherosclerotic heart disease $(5 \%)$.

- Table 2 showed the results of measurements of the two groups of patients with respect to tissue Doppler measurement and long-axis left ventricular function. Group A (number $=89$ ) consisted of patients who had no MACE and revascularization procedures in the subsequent period of follow-up (6months). Group B (number $=11$ ) consisted of patients who had revascularization procedures and/or significant CAD documented by invasive coronary angiography 
Table 1 Baseline demographic and clinical data $(n=100)$

\begin{tabular}{|l|l|}
\hline Characteristic & Value \\
\hline Age, years, mean \pm SD & $48 \pm 10$ \\
\hline Sex & $65(65)$ \\
\hline Male & $35(35)$ \\
\hline Female & $76(76)$ \\
\hline Race & $24(24)$ \\
\hline Chinese & $58(58)$ \\
\hline Caucasians & $62(62)$ \\
\hline Hypertension & $5(5)$ \\
\hline Dyslipidemia & $5(5)$ \\
\hline Family history of coronary disease & $15(15)$ \\
\hline Diabetes mellitus & $40(40)$ \\
\hline Smoking & \\
\hline Left ventricular hypertrophy &
\end{tabular}

Abbreviation: SD, standard deviation.

Note: Data presented as $n(\%)$ unless otherwise indicated.

\pm pressure flow wire/intravascular ultrasound interrogation in the subsequent period of follow-up ( 6 months). All patients in Group B had CT angiography and an invasive coronary angiogram. Nine patients had revascularization procedures after obstructive CAD was found. Out of the 11 patients in Group B, 2 patients had one-vessel CAD, 8 patients had twovessel $C A D$, and 1 patient had three-vessel CAD. One patient had coronary artery bypass grafting, eight patients had percutaneous revascularization procedures, and two other patients elected to continue medical management despite obstructive CAD after an invasive coronary angiogram was done. None of the patients in Group B suffered an MACE in the

Table 2 Tissue Doppler and long-axis function results among Group A and Group B patients

\begin{tabular}{|l|l|l|}
\hline & Group A & Group B \\
\hline Number & 89 & 11 \\
\hline $\begin{array}{l}\text { Ea (maximum early } \\
\text { diastolic velocities) (cm/s) }\end{array}$ & $11.4 \pm 3.5$ & $\begin{array}{l}8 \pm 1.1 \\
(p=0.002)\end{array}$ \\
\hline $\begin{array}{l}\text { Sa(maximum systolic } \\
\text { velocities)(cm/s) }\end{array}$ & $11.36 \pm 3.1$ & $\begin{array}{l}8.9 \pm 2.4 \\
(p=0.012)\end{array}$ \\
\hline $\begin{array}{l}\text { Ea/Aa (ratio of maximum } \\
\text { early diastolic velocities } \\
\text { and maximum late } \\
\text { diastolic velocities) }(\mathrm{cm} / \mathrm{s})\end{array}$ & $1.34 \pm 2.23$ & $\begin{array}{l}0.71 \pm 0.15 \\
(p=0.01)\end{array}$ \\
\hline \begin{tabular}{l} 
Long-axis excursion $(\mathrm{cm} / \mathrm{s})$ \\
\hline
\end{tabular} & $1.64 \pm 0.29$ & $\begin{array}{l}1.32 \pm 0.12 \\
(p<0.01)\end{array}$ \\
\hline
\end{tabular}

Abbreviations: CAD, coronary artery disease; MACE, major adverse cardiac event.

Note: Group A-patients who did not have MACE and/or revascularization procedures in the following 6 months after the cardiac testing. Group Bpatients who had revascularization procedures and/or significant $C A D$ shown on invasive coronary angiography in the following six months after the cardiac testing.
Table 3 Sensitivity, specificity, PPV, NPV, and accuracy for segmental wall motion analysis in predicting significant CAD shown on coronary angiography and/or future revascularization

\begin{tabular}{|l|l|c|}
\hline Abnormal segmental wall motion & Positive & Negative \\
\hline Significant CAD/revascularization + & 8 & 3 \\
\hline Significant CAD/revascularization - & 18 & 71 \\
\hline
\end{tabular}

Abbreviations: CAD, coronary artery disease; NPV, negative predictive value; PPV, positive predictive value.

Note: Sensitivity $=73 \%$, PPV $=31 \%$; specificity $=80 \%, N P V=96 \%$; accuracy $=79 \%(p<0.001)$.

subsequent 6 months. Two other patients in Group A underwent coronary angiography which showed nonobstructive CAD evidenced by the angiogram \pm pressure flow wire/ intravascular ultrasound interrogation.

- Table 2 showed that low Aa, Sa, and Ea/Aa values and a depressed long-axis function were associated with obstructive CAD and subsequent revascularization.

- Table 3 shows the sensitivities, specificities, positive predictive values, negative predictive values, and accuracies for abnormal wall motion analysis to predict significant CAD on invasive coronary angiography and/or revascularization procedures (Group B). One should note that using abnormal wall motion analysis as the sole tool, the sensitivity (73\%) and positive predictive value (31\%) were somewhat limited but the specificity (80\%) and the negative predictive value (96\%) were still acceptable with a fair degree of accuracy (79\%).

An E/Ea ratio more than 10 (-Table 4) did not predict patients who had significant CAD on coronary angiography with subsequent revascularization procedures with inadequate sensitivity (55\%), specificity (78\%), accuracy (75\%), and a poor positive predictive value (24\%).

However, the combined novel criteria (-Table 5) (i.e., classifying a case as positive if three out of the following four criteria were fulfilled) offered a very high accuracy for predicting obstructive CAD and future revascularization with a high sensitivity (100\%), high specificity (87\%), high negative predictive value (100\%), and a very good accuracy (88\%), though the positive predictive value was limited (48\%):

1. Abnormal segmental wall motion shortly after peak stress

2. Ee wave after peak stress less than $10 \mathrm{~cm} / \mathrm{s}$ andEe/Aa ratio after peak stress less than 1

Table 4 Sensitivity, specificity, PPV, NPV, and accuracy for an elevated estimated left ventricular filling pressure (E/Ea $>10)$ in predicting significant CAD shown on invasive coronary angiography and/or future revascularization

\begin{tabular}{|l|l|l|}
\hline E/Ea & $>10$ & $\leq 10$ \\
\hline Significant CAD/revascularization + & 6 & 5 \\
\hline Significant CAD/revascularization - & 20 & 69 \\
\hline
\end{tabular}

Abbreviations: CAD, coronary artery disease; NPV, negative predictive value; PPV, positive predictive value.

Note: Sensitivity $=55 \%$, PPV $=23 \%$; specificity $=78 \%, N P V=93 \%$, accuracy $=75 \%(p=0.054)$. 
Table 5 Sensitivity, specificity, PPV, NPV, and accuracy for novel combined criteria ${ }^{a}$ in predicting significant CAD on invasive coronary angiography and/or future revascularization

\begin{tabular}{|l|l|l|}
\hline Novel combined criteria & Positive & Negative \\
\hline Significant CAD/revascularization + & 11 & 0 \\
\hline Significant CAD/revascularization - & 12 & 77 \\
\hline
\end{tabular}

Abbreviations: CAD, coronary artery disease; NPV, negative predictive value; PPV, positive predictive value.

Note: Sensitivity $=100 \%$, PPV $=48 \%$; specificity $=87 \%$, NPV $=100 \%$; accuracy $=88 \%(p<0.001)$.

${ }^{\mathrm{a}}$ Novel combined criteria classified a case as positive if three out of the following four criteria were fulfilled:

1. Abnormal segmental wall motion shortly after peak stress.

2. Ee wave after peak stress less than $10 \mathrm{~cm} / \mathrm{s}$ and Ee/Aa ratio after peak stress less than 1.

3. Sm wave after peak stress less than $10.5 \mathrm{~cm} / \mathrm{s}$.

4. Abnormal long-axis left ventricular function after peak stress: amplitude of long-axis shortening less than $1.35 \mathrm{~cm}$ in female and less than $1.55 \mathrm{~cm}$ in male.

3. Sm wave after peak stress less than $10.5 \mathrm{~cm} / \mathrm{s}$

4. Abnormal long-axis left ventricular function: amplitude of long-axis shortening less than $1.35 \mathrm{~cm}$ in female and less than $1.55 \mathrm{~cm}$ in male.

\section{Discussion}

Clinical studies ${ }^{6,7}$ have shown that the addition of TDI to measure resting or poststress velocities of various myocardial segments of LV or LV diastolic function as an adjunct tool may help in the diagnosis of significant CAD. Its use may be valuable even in cases where segmental wall motion abnormalities are not yet present, like in patients with poor echocardiographic windows or in patients with suboptimal exercise response. Mechanistically, an ischemic cascade starts with bloodflow heterogeneity, followed by diastolic dysfunction (including relaxation abnormalities), then localized systolic dysfunction, ischemic electrocardiographic changes, and finally chest discomfort/angina equivalent symptoms. Because pseudonormalization of Doppler waveforms does not usually occur in TDI analysis of diastolic dysfunction, an Ea/Aa ratio less than 1 almost always implies the presence of diastolic dysfunction, from relaxation abnormalities to restrictive filling abnormalities. Ea wave, however, decreases in proportion to different degrees of diastolic dysfunction. Since diastolic dysfunction occurs early in the ischemic cascade, it is possible that CAD patients with suboptimal exercise tolerance may show an early sign of diastolic dysfunction by TDI technique, thereby avoiding the potential false-negative testing.

Long-axis shortening of LV during dobutamine stress was considered by some investigators as the best predictor of CAD. ${ }^{5}$ Abnormal long-axis function was more sensitive than wall motion change in detecting single-vessel CAD (sensitivity $94 \%$ vs. $75 \%$ ). Subendocardial long-axis fibers are more susceptible to ischemia. Therefore, long-axis dysfunction occurs early in ischemia (compensated by the circumferentially arranged radial fibers), sometimes even without segmental wall motion abnormalities. Recovery of long-axis function is also slower than circumferential radial fibers after an episode of ischemia. This might explain why it is useful as an adjunctive tool when stress-induced wall motion abnormalities resolve quickly before being documented by poststress images. Absence of long-axis dysfunction and wall motion abnormalities is highly predictive of an absence of significant CAD.

Despite the additional advantages of TDI and long-axis function, the routine use of the above techniques has not been adopted in a community setting, mostly due to the additional time involved, the potential cumbersome acquisition protocol, and the lack of consensus on the value of these techniques.

The current study did confirm the feasibility of adding simplified TDI and long-axis function to the established stress echocardiography protocol (using wall motion analysis as the sole criterion). The additional value of the novel combined criteria improved the sensitivity, specificity, negative predictive value, and accuracy of the test (from $73 \%$ to $100 \%, 80 \%$ to $87 \%, 96 \%$ to $100 \%$, and $79 \%$ to $88 \%$, respectively).

With $100 \%$ sensitivity and $100 \%$ negative predictive value of the testing using the combined criteria, it is highly possible that in a low-to-intermediate risk population, no more cardiac testing needs to be performed if a negative stress echocardiographic result is obtained using the novel method, thus avoiding the invasiveness and the potential side effects (such as radiation exposure and contrast allergy) of invasive coronary angiography and CT angiography. Further larger studies need to be performed to validate the above point.

\section{Limitations}

First, the present study was retrospective; therefore, it suffers from the limitations conferred by its retrospective style. The number of subjects studied was small, so statistical errors may have been introduced. The sensitivity of the stress echocardiographic test using the wall motion analysis in the current study was limited (73\%), much lower than the sensitivity published in literature (80-85\%). The reason may be due to the fact that the number of subjects was small. Also, several patients had poor echocardiographic windows, and some patients did not achieve an adequate exercise capacity, rendering submaximal stress and false-negative test results. In the three patients who had false-negative testing by using wall motion analysis, one patient had one-vessel CAD andthe other two patients had suboptimal exercise tolerance. Interestingly, the novel method using the combined criteria very much likely picked up early ischemic changes, and so it correctly identified all three patients as positive, avoiding a potentially adverse cardiac outcome.

The positive predictive value of the novel method by using the combined criteria was limited (48\%), which was reasonable in a low-risk population with a low prevalence of the disease. If the study were repeated in a population with a higher prevalence of the disease, the positive predictive value would likely improve. A high false positive rate using the combined criteria may be also due to the high prevalence of diastolic dysfunction with the presence of left ventricular hypertrophy and hypertension in the current study cohort. 


\section{Conclusion}

Based on the above analysis, it was demonstrated that by using the novel combined criteria in stress echocardiography, the accuracy of the stress echocardiography was improved and a negative result was associated with a favorable clinical outcome in the subsequent 6 months (i.e., classifying a case as positive if three out of the following four criteria were fulfilled):

1. Abnormal segmental wall motion shortly after peak stress

2. Ee wave after peak stress less than $10 \mathrm{~cm} / \mathrm{s}$ and Ee/Aa ratio after peak stress less than 1

3. Sm wave after peak stress less than $10.5 \mathrm{~cm} / \mathrm{s}$

4. Abnormal long-axis left ventricular function: amplitude of long-axis shortening less than $1.35 \mathrm{~cm}$ in female and less than $1.55 \mathrm{~cm}$ in male

In addition, in a low-to-intermediate risk population, a negative stress echocardiogram using the novel combined criteria has an excellent negative predictive value (100\%) and possibly warrants no further invasive cardiac testing. Further larger prospective studies are required to validate the above point.

\section{References}

1 Fleischmann KE, Hunink MG, Kuntz KM, Douglas PS. Exercise echocardiography or exercise SPECT imaging? A meta-analysis of diagnostic test performance. JAMA 1998;280(10):913-920
2 Pellikka PA, Nagueh SF, Elhendy AA, Kuehl CA, Sawada SG; American Society of Echocardiography. American Society of Echocardiography recommendations for performance, interpretation, and application of stress echocardiography. J Am SocEchocardiogr 2007;20(9):1021-1041

3 Quiñones MA, Verani MS, Haichin RM, Mahmarian JJ, Suarez J, Zoghbi WA. Exercise echocardiography versus 201Tl single-photon emission computed tomography in evaluation of coronary artery disease. Analysis of 292 patients. Circulation 1992;85(3): 1026-1031

4 Armstrong WF, Zoghbi WA. Stress echocardiography: current methodology and clinical applications. J Am CollCardiol 2005; 45(11):1739-1747

5 Mishra MB, Lythall DA, Chambers JB. A comparison of wall motion analysis and systolic left ventricular long axis function during dobutamine stress echocardiography. Eur Heart J 2002;23(7): 579-585

6 Agarwal R, Gosain P, Kirkpatrick JN, et al. Tissue Doppler imaging for diagnosis of coronary artery disease: a systematic review and meta-analysis. Cardiovasc Ultrasound 2012;10:47

7 Mädler CF, Payne N, Wilkenshoff U, et al; Myocardial Doppler in Stress Echocardiography (MYDISE) Study Investigators. Non-invasive diagnosis of coronary artery disease by quantitative stress echocardiography: optimal diagnostic models using off-line tissue Doppler in the MYDISE study. Eur Heart J 2003;24(17):1584-1594

8 Tobis J, Azarbal B, Slavin L. Assessment of intermediate severity coronary lesions in the catheterization laboratory. J Am CollCardiol 2007;49(8):839-848

9 Mintz GS, Nissen SE, Anderson WD, et al. American College of Cardiology Clinical Expert Consensus Document on Standards for Acquisition, Measurement and Reporting of Intravascular Ultrasound Studies (IVUS). A report of the American College of Cardiology Task Force on Clinical Expert Consensus Documents. J Am CollCardiol 2001;37(5):1478-1492 